drugs are often prescribed alongside statins for cardiovascular disease, but no changes in risk estimates were observed after adjustment for their use in this analysis.

Although further studies are needed before clinical implications can be drawn, this study adds to the increasing evidence that statins might have anti-cancer properties. These findings will be reassuring for the many patients who take statins regularly.

Original article Fortuny J et al. (2006) Statin use and risk of lymphoid neoplasms: results from the European CaseControl Study EPILYMPH. Cancer Epidemiol Biomarkers Prev 15: 921-925

\section{Bcl-6 protein not a prognostic indicator in patients with DLBCL receiving $\mathrm{R}-\mathrm{CHOP}$ therapy}

The addition of rituximab (R) to CHOP (cyclophosphamide, doxorubicin, vincristine, and prednisone) is a new strategy in the treatment of diffuse, large B-cell lymphomas (DLBCLs). Researchers from the US have reported that $\mathrm{R}-\mathrm{CHOP}$ could potentially be limited to patients who do not express the Bcl-6 protein, a marker of germinal center origin.

$\mathrm{Bcl}-6$ expression is predictive of favorable outcome in patients with DLBCL treated with conventional CHOP. Winter et al. aimed to determine whether this biomarker retains its prognostic significance with R-CHOP. Their analysis formed part of a US Intergroup trial, which randomized patients with DLBCL aged 60 years or over to receive $\mathrm{CHOP}$ or $\mathrm{R}-\mathrm{CHOP}$. Tissue samples from 199 patients (CHOP 46\%, R-CHOP 54\%) were analyzed for $\mathrm{Bcl}-6$ expression.

The 2-year failure-free and overall survival rates were higher in Bcl-6-positive patients treated with $\mathrm{CHOP}$ than in similarly treated cases who were Bcl-6-negative. Conversely, Bcl-6 status did not affect the failure-free or overall survival of patients in the R-CHOP group. In the multivariate analysis, the relative risk of treatment failure and death from DLBCL was significantly lower for Bcl-6-negative patients treated with $\mathrm{R}-\mathrm{CHOP}(P<0.001)$ than for those treated with $\mathrm{CHOP}$ alone. For Bcl-6positive patients, the treatment regimen did not significantly impact the long-term outcome. The authors conclude that R-CHOP does not benefit patients over the age of 60 whose DLBCLs express Bcl-6, and that the prognostic significance of this marker is lost when the R-CHOP combination is used.

Original article Winter JN et al. (2006) Prognostic significance of Bcl-6 protein expression in DLBCL treated with CHOP or R-CHOP: a prospective correlative study. Blood 107: 4207-4213

\section{Genetic profiling could identify patients at risk of relapse of colorectal cancer}

Patients who present with early-stage colorectal cancer without metastasis (Dukes' stage A or B) typically undergo surgery without adjuvant therapy. Despite having apparently low-risk disease, $10-40 \%$ of these patients relapse after surgery, and up to $50 \%$ die from complications or metastasis. Molecular markers that can predict relapse in patients with early-stage colorectal cancer are being investigated, but previous studies analyzed only a few candidate markers.

Al-Mulla and colleagues' study enrolled 70 patients with early-stage, nonmetastatic colorectal cancer. Tumor samples from 27 patients who relapsed after surgery were compared with samples from 43 patients who did not relapse during follow-up (range 2-9 years). The authors used comparative genomic hybridization to identify chromosomal aberrations in a total of 61 tumors; their findings were confirmed by DNA microarray analysis of 10 tumors.

This genome-wide analysis identified many abnormalities that correlated with relapse of early-stage colorectal cancer after surgery, including loss of chromosomes $1 p, 4,5 q, 8 p$, $9 p, 14 q$, and 18p, and gain of chromosomes 1q, 8q, and 13q. Notably, samples from patients who relapsed were more likely to have multiple aberrations (e.g. loss of both $8 p$ and 18q, and loss of both 4 and $14 q$ ). These specific multiple aberrations were associated with markedly short survival after surgery. Loss of chromosome 4 independently predicted poor survival.

The same team has recently used an unrelated technique (analysis of highly polymorphic microsatellite markers) to narrow down the deletions on chromosomes 4 and 14 to specific regions.

Original articles Al-Mulla F et al. (2006) Genetic profiling of stage I and II colorectal cancer may predict metastatic relapse. Mod Pathol 19: 648-658

Al-Mulla F et al. (2006) Metastatic recurrence of early-stage colorectal cancer is linked to loss of heterozygosity on chromosomes 4 and 14q. J Clin Pathol 59: 624-630 